\title{
THREE-DIMENSIONAL DEFORMATION OF WARP-KNITTED SPACER FABRICS UNDER TENSILE LOADING
}

\author{
Wan Sun ${ }^{1,2}$, Xuhong Miao ${ }^{1,3, *}$, Raji Rafiu King ${ }^{1}$, Pibo Ma $^{1,3}$ \\ ${ }^{1}$ Engineering Research Center for Knitting Technology, Ministry of Education, Jiangnan University, Wuxi 214122, China; \\ ${ }^{2}$ Key Laboratory of Eco-textiles, Ministry of Education, Jiangnan University, Wuxi 214122, China; \\ ${ }^{3}$ International Joint Research Laboratory for Novel Knitting Structural Materials, Jiangnan University, Wuxi 214122, China \\ *Corresponding author: miaoxuhong@163.com
}

\begin{abstract}
:
This paper puts forward a new method for measuring the three-dimensional deformation of warp-knitted spacer fabrics under tensile stress. The three-dimensional deformation mechanisms of warp-knitted spacer fabrics have been analyzed using stress-strain curves. Poisson's ratio of the three-dimensional deformation has also been analyzed. The stress-strain curves obtained for tests in the warp-ward direction and weft-ward direction show a characteristic initial large deformation, followed by minimal-to-no deformation. The stress-strain curves obtained for tests in the thickness direction exhibit different characteristics due to the differences in stretch directions. In the weft-ward direction, the curve shows an approximate linear change with minimal strain. In the warp-wise direction, the curve shows a large stress with small strain, and subsequently, a small stress yielded a large strain. During the stretching process, the surface deformation perpendicular to the direction of tensile force is greater than the tensile deformation, and the deformation in the thickness direction is also minimal compared to that in the direction of the tensile deformation.
\end{abstract}

\section{Keywords:}

warp knitted, spacer fabrics, three-dimensional deformation

\section{Introduction}

Warp-knitted spacer fabrics have a special three-dimensional (3D) structure, which makes them suitable for a wide range of applications, including for clothing, shoe material, cushion applications, filter material, as well as medical and health care products. Their utility is enhanced by virtue of their excellent water vapor permeability, compressive elasticity, sound insulation, and overall structural moldability [1-3]. Although warp-knitted spacer fabrics constitute a recent development as far as knitting technology is concerned, they have become popular materials for current research and development. At present, research on the performance of warp-knitted spacer fabrics is mainly focused on compression performance [46], stab resistance performance [7], and bending property [8]. There is little research on the tensile properties and 3D deformation of warp-knitted spacer fabrics. In terms of fabric deformation under tensile stress, scholars' present research efforts are focused on woven fabric, weft-knitted fabric, and warp-knitted fabric. The anisotropy of tensile properties [9, 10], force model [11], biaxial stretching stress-strain curve [12, 13], the influence of the stretching direction on the breaking strength and the elongation at break [14], and so on are the major areas of research. These research analyses rarely involve the deformation that is perpendicular to the stretching direction when the fabric is stretched. Though the research on Poisson's ratio of materials [15-17] focuses on the deformation that either is in the same orientation or is perpendicular to the stretching direction, research works rarely involve the relationship between the vertical stretching direction and the tensile stress in the deformation process. In this paper, the relationship between the $3 \mathrm{D}$ size variation and the tensile stress is studied by proposing a new test method.

\section{Experimental}

Until now, there has not been any record of research on the 3D warp-knitted spacer fabric's deformation; therefore, there are no corresponding measurement standards and test methods. In order to study the 3D deformation of warp-knitted spacer fabrics, this paper presents a relatively accurate test method.

\subsection{Materials and Methods}

The samples were knitted using double needle bar warpknitting machine (Karl Mayer, RD6N). The machine has six guide bars. Tensile tests were carried out using Ningbo Kewei multifunctional electronic fabric strength testing machine (Ningbo Kewei Textile Instrument Company Ltd, PRC). Digital cameras were manufactured by Canon.

\subsection{Production of Samples}

The structures of warp-knitted spacer fabrics are varied. Three typical kinds of warp-knitted spacer fabrics have been used in this study, as shown in Figure 1.

The production specifications of the samples are also shown in Table 1. The two surface layers of Sample A have been varied, 


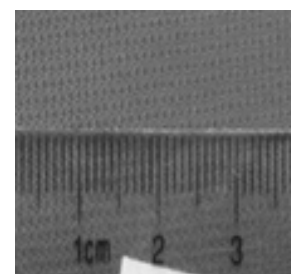

(a)

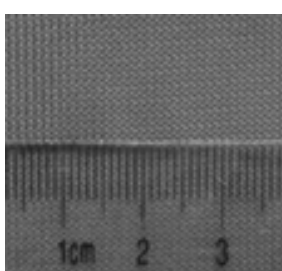

(b)

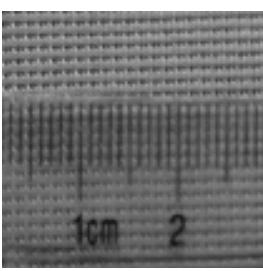

(c)

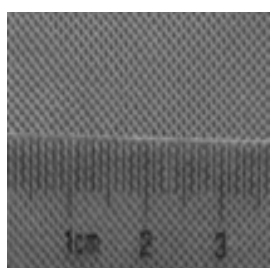

(d)

Figure 1. The surface layers of samples: (a) first surface of Sample A; (b) second surface of Sample A; (c) Sample B; (d) Sample C

Table 1. Fabric production parameters

\begin{tabular}{|c|c|c|c|}
\hline Sample & Structure & Threading & Yarn count \\
\hline$A$ & $\begin{array}{l}\text { GB1:1-0-0-0/2-3-3-3// } \\
\text { GB2:1-2-2-2/1-0-0-0// } \\
\text { GB3:1-0-1-2/2-3-2-1// } \\
\text { GB4:1-1-1-2/1-1-1-0// } \\
\text { GB5:1-1-1-0/1-1-1-2// }\end{array}$ & $\begin{array}{l}\text { Full } \\
\text { Full } \\
\text { Full } \\
\text { Full } \\
\text { Full }\end{array}$ & $\begin{array}{c}\text { 8.3tex 36f Polyester } \\
\text { 8.3tex 36f Polyester } \\
\text { 3.3tex Polyester } \\
\text { 8.3tex 36f Polyester } \\
\text { 8.3tex 36f Polyester }\end{array}$ \\
\hline B & $\begin{array}{l}\text { GB1:1-0-0-0/1-2-2-2// } \\
\text { GB2:1-2-2-2/1-0-0-0// } \\
\text { GB3:1-0-1-2/2-3-2-1// } \\
\text { GB4:1-1-1-2/1-1-1-0// } \\
\text { GB5:1-1-1-0/1-1-1-2// }\end{array}$ & $\begin{array}{l}\text { Full } \\
\text { Full } \\
\text { Full } \\
\text { Full } \\
\text { Full }\end{array}$ & $\begin{array}{c}\text { 11.1tex 36f Polyester } \\
\text { 11.1tex 36f Polyester } \\
\text { 3.3tex Polyester } \\
\text { 11.1tex36f Polyester } \\
\text { 11.1tex 36f Polyester }\end{array}$ \\
\hline $\mathrm{C}$ & $\begin{array}{l}\text { GB1:1-0-0-0/2-3-3-3// } \\
\text { GB2:2-3-3-3/1-0-0-0// } \\
\text { GB3:1-0-1-2/2-3-2-1// } \\
\text { GB4:2-2-2-3/1-1-1-0// } \\
\text { GB5:1-1-1-0/2-2-2-3// }\end{array}$ & $\begin{array}{l}\text { Part set threading } \\
\text { Part set threading } \\
\text { Full } \\
\text { Part set threading } \\
\text { Part set threading }\end{array}$ & $\begin{array}{c}\text { 8.3tex 36f Polyester } \\
\text { 8.3tex 36f Polyester } \\
\text { 3.3tex Polyester } \\
\text { 8.3tex 36f Polyester } \\
\text { 8.3tex 36f Polyester }\end{array}$ \\
\hline
\end{tabular}

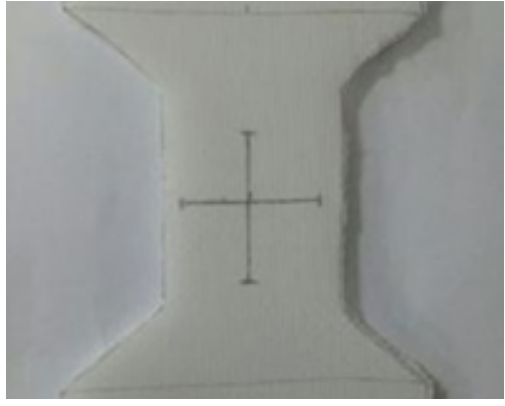

Figure 2. Presentation of sample markings

using tricot and reverse locknit, respectively. Those of Sample $B$ are plain structured, and the two surfaces of Sample $C$ show a mesh structure.

The experimental samples were trimmed into trapezoid shapes with widths of $50 \mathrm{~mm}$. In order to avoid the influence of the chuck on the dimensional deformation measurements at the fabric edge, a crosshair of $4 \mathrm{~cm} \times 4 \mathrm{~cm}$ was drawn in the middle

Table 2. Fabric structure characterization parameters

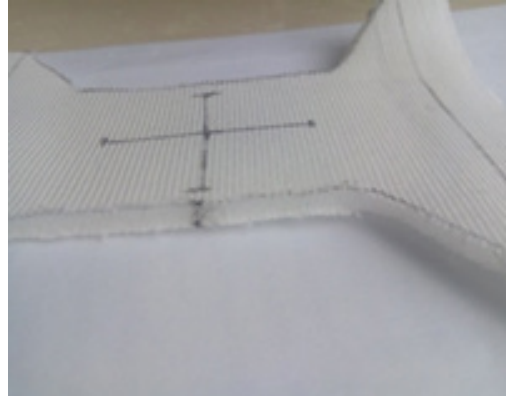

(b)

\begin{tabular}{|c|c|c|c|c|c|}
\hline Sample & $\begin{array}{c}\text { Wale per } \\
\text { centimeter }\end{array}$ & $\begin{array}{c}\text { Course per } \\
\text { centimeter }\end{array}$ & Thickness & CV\% & $\begin{array}{c}\text { Weight per square } \\
\text { meter }\end{array}$ \\
\hline A & 10.6 & 11.5 & 3.89 & 1.1 & 334.2 \\
\hline B & 10.2 & 15.1 & 2.85 & 0.95 & 303.5 \\
\hline C & 9.4 & 14.2 & 2.10 & 0.83 & 323.1 \\
\hline
\end{tabular}

position of the trapezoidal specimen, as shown in Figure 2(a), to measure the size change in the horizontal and vertical stretching directions. The same has been done in the spacer interval to measure the deformation in that section, as shown in Figure 2(b).

Five samples for each fabric type were experimented on, and their averages were computed as the final results. 


\section{$\underline{2.3 \text { Measurement method }}$}

The experimental conditions were as follows: laboratory temperature $-18^{\circ} \mathrm{C}$, relative humidity $-30 \%$. The tensile test was based on the GB/T 3923.1-2013 standard "fabric tensile strength, breaking strength and elongation at break determination" methods. The parameters for the multifunctional electronic fabric strength instrument were set as follows: clamp distance of $100 \mathrm{~mm}$, chuck moving speed of $100 \mathrm{~mm} / \mathrm{min}$, and sample pretension of $2.0 \mathrm{~N}$. Digital cameras were placed on the front and side of the measuring instrument, and the lens was kept perpendicular to the ground and in close contact with the fabric. The height of the cameras was adjusted to ensure that the screen was aligned with the center of the fabric.

The tensile test and the video recording were set to run concurrently until the fabric reached its breaking point and then the video recording was stopped. Taking the warp tensile test as an example, the 3D test method is described in detail as follows. Deformation of the specimen in the vertical stress direction was measured using a front view of the shot, as shown in Figure 3(a).

The deformation in the stretching direction was determined by the strength tester's elongation values. The deformation at the spacer section or thickness direction was measured based on the side view snapshot, as shown in Figure 3(b). The tensile strength of the specimen was recorded by the tensile meter. This test method had a time delay. The beginning of the camera recording and the start of the stretching had been not synchronized. The tensile tester begins stretching with a "beep" tone. The tone in the two videos can be heard clearly, so the time of hearing the tone is defined as $t=0$. The specific steps are as follows:
Adobe Premiere Pro CS6 software was used to process the recorded videos, and the times $t_{1}$ and $t_{2}$ were set frame by frame to enable playback.

Moreover, $t_{1}, t_{2}$ represent video start times and $t=0$ also denotes the stretching of fabric. A single frame of picture from the video was extracted at intervals of $\mathrm{D} t=0.1 \mathrm{~s}$ until the fabric broke. Values for the degrees of deformation in both the vertical stress direction and the thickness direction were recorded.

The tensile data recorded by the tensile strength tester included the tensile force $F$, the stretching time $t$, the tensile length $I$, and other parameters. The values of $F$ and $I$ are recorded when $t=0.1 n(n=0,1,2,3 \ldots)$, where the tensile length $I$ is the dimensional change of the fabric in the stretching direction.

The tensile data values in the vertical stretching direction, the thickness section, and $F$ were all aggregated to obtain the strain curves in the three directions of the warp-knitted spacer fabric.

\section{Results and discussion}

Samples A, B, and C were subjected to warp- and weft-directional tensile tests according to the above-mentioned methods, and the images and data were processed to produce a stress-strain curve. The abscissa is the stress value in the tensile direction of the fabric. The fabric stress is presented as follows:

$$
\mathrm{s}=F M
$$

where $F$ is the load (in Newtons) on the fabric, and $W$ is the width (in centimeters) of the section of the fabric. The ordinate is the deformation, $\mathrm{e}=\left|\left(I-I_{0}\right) / I_{0}\right|$.
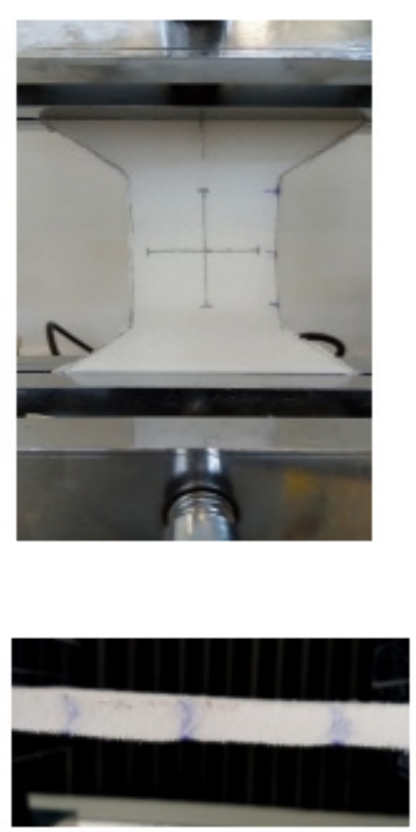
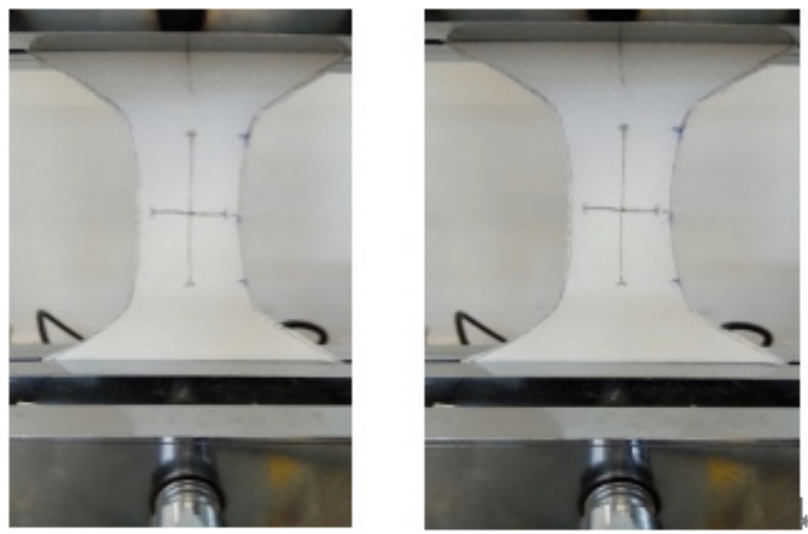

(a)
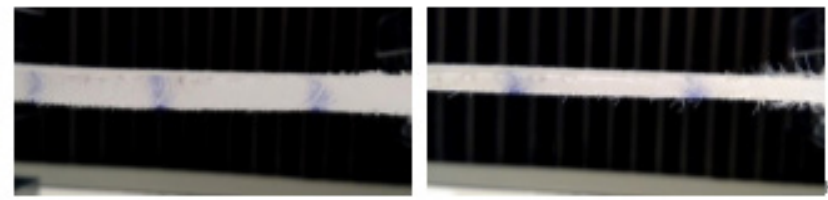

(b)

Figure 3. Images of samples showing the orientations during the tensile deformation process: (a) front view of sample; (b) side view of samples 
$I_{0}$ is the original size of the fabric (in centimeters), and $I$ is the size of the fabric after stretching (in centimeters).

\subsection{Analysis of the Stress-Strain Curves}

From the stress-strain curves of samples A, B, and C in the weft-wise and warp-wise directions, as shown in Figure 4, it can be seen that a small stress leads to large strain initially; with the tensile process continuing, large stress just results in small strain. This characteristic is the same as that of conventional fabrics, although warp-knitted spacer fabrics have a 3D structure. Not much explanations are provided here.

A comparison of the warp-wise stress-strain curves of specimens $A, B$, and $C$, as shown in Figure 4, shows that Sample $B$ had the highest breaking stress, followed by $C$ and $A$ in that order. In the weft-wise direction, the breaking stress of Sample B was also the largest, with samples A and C following as the second and third, respectively. The breaking stress of the fabric is affected by many factors, such as yarn threading, structure, fabric density, and so on. According to the experimental sample specifications, yarn of the Sample B was the coarsest and the weft density of Sample B was the highest.

The coarseness of the yarn for samples $C$ and $A$ was the same, but the weft density of $C$ was higher than that for A. When the samples $A / B / C$ were stressed in the warpwise direction, more yarns in Sample $B$ bore the tension. Furthermore, the yarn of Sample B was the thickest, thus informing its highest breaking stress. Although Sample C was partly threaded, the weft density was higher than that for Sample A. The yarns of samples $A$ and $C$ both had the same thickness; hence, Sample $C$ had a bigger breaking stress compared to Sample A. In the weft-wise orientation, the weft density of Sample A was higher than that of Sample $B$; however, the breaking stress of Sample B was bigger than that for Sample A. This is because the yarn of Sample B was thicker than the yarn of Sample A. Sample A had a higher weft density compared to Sample C; however, both samples had yarns of equal thickness. This, therefore, informs the bigger breaking stress of Sample A compared to Sample C. These permutations thereby clarify the impact of same or differing yarn coarseness levels on structural density, whether high or low, as well as the impact of the corresponding stress magnitude. If the densities are the same, the thicker yarn tends to deliver the biggest breaking stress. In addition, the stress-strain curve shows that the tensile deformation of different structures in the warp-ward and weft-ward directions is different. The initial modulus of Sample B and Sample $C$ in the warp-ward direction was larger than that in the weft-ward direction. It indicated that samples $B$ and $A$ are more prone to deformation in the weft-ward direction. Sample A is contrary to samples B and C. Therefore, some fabrics are easily deformed by warp-stretching and some are more easily deformed in the weft direction. This, therefore, suggests that the degree of difficulty of deformation in a particular direction can determine the application and end use of the structures.

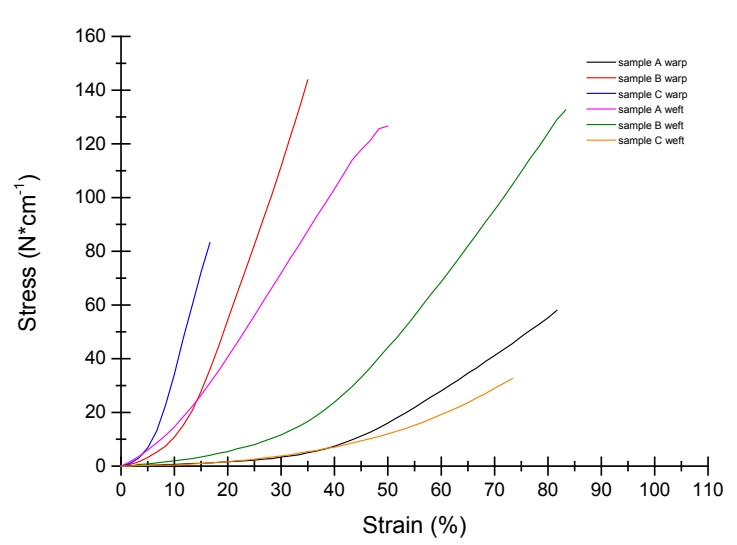

Figure 4. Stress-strain curve of sample

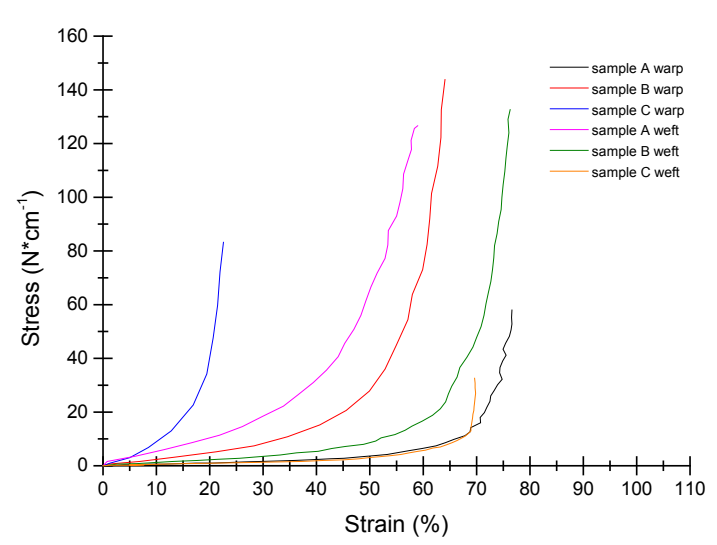

Figure 5. Stress-strain relations showing vertical stretch direction deformations

\subsection{Analysis of Stress-Strain Curve - Vertical Stretch Deformation}

Figure 5 shows the results of the wale-wise and the coursewise stresses on the fabrics and their accompanying lateral deformations. It can be seen that a small initial stress resulted in large fabric deformation; however, as the stress was increased, the deformation tended to be marginal. And with subsequent increases in tensile stress, the deformation gradually tended to remain unchanged. The loop transfer accounts for this phenomenon. At the initial stage of stretching in the warp-ward direction (in the weft-ward direction), the fabric is more relaxed, so there are large gaps between the loops. By extending the fabric, the weft (warp) loop is transferred more easily, and the rate of extension is quite large. With the increase in tensile force, weft (warp) loop transfer or extension is exhausted. Yarn elongation becomes very difficult when the fabric is in a tense state. Therefore, deformation of fabric in the weft-wise plane does not change substantially.

A comparison of the samples $A, B$, and $C$ in both the warpwise and weft-wise stretching directions using the stress-strain curves reveals that for the maximum strain of the specimen, deformation perpendicular to the stretching direction is also the biggest, which proves that loops are transferred when the fabric is stretched. 


\subsection{Analysis of Stress-Strain Curve - Deformation of the Spacer Interval}

The stress-strain curves of the samples representing the deformation at the spacer intervals, as sketched in Figure 6 , show the different characteristics due to differences in the stretching direction. With regard to weft-wise stretching, the curves exhibit an approximately linear variation between the stress and the strain in the thickness or spacer interval direction, and the strain range is small. With regard to warpwise stretching in the thickness direction, a small deformation resulted during large stress. Subsequently, large deformation is also produced by small stress.

The spacer yarns in the fabric are warp fed, and so when the weft is stretched, the main load of the force on the fabric tends to be concentrated on the two surfaces. The intermediate spacer layer is not directly subjected to stress; hence, its deformation is small. The curve as such exhibits an approximately linear change in the thickness direction with a small strain range. When the warp-knitted fabric is stretched in the warp-wise direction, the layer loops, and the loops formed by the spacer yarns on the surface bear the tension. In the initial stage of stretching, the surface loop is looser. The spacer interval filament loops bear the small force. Therefore, at the initial stage of stretching, the deformation of the spacer yarn is small, resulting in a large stress with small deformation characteristics. With the increase in tension, however, the surface loop structure is transformed to a tight state, and the continuous force on them is enhanced. Therefore, the spacer yarn is elongated to produce large deformation, a confirmation of the characteristic small stress-large deformation.

In addition, the deformation of the warp-knitted fabric in the thickness direction is generally smaller than that of the other two directions. This is mainly because the weft and warp deformations of the fabric are directly caused by the tensile force, so the deformation is lager. And the dimensional change in the thickness direction is due to the change in the length of the spacer yarn due to the movement of the surface layer loop. Furthermore, the inclination of the interval filament is increased, resulting in a small change in the thickness direction.

\subsection{Poisson's Ratio of Warp-Knitted Fabric}

Poisson's ratio is defined as $E i j=(-e j) / e i$, where ej denotes the lateral deformation during vertical stress and ei is the elongation strain in the stretching direction. Further, ej1 represents the strain of the stretching direction of the vertical surface layer, $e_{j 2}$ also denotes the strain of thickness, $E i j_{1}$ is the Poisson's ratio of the warp-knitted spacer surface, Eij ${ }_{2}$ is the Poisson's ratio of the warp-knitted spacer thickness. Based on the experimental data, two curves are drawn. They are the ej1-ei curve and the ej2-ei curve.

It can be seen from Figure 7 that the Poisson's ratio curve of the spacer surface shown in Figure 7(a) is different from the Poisson's ratio curve in the thickness direction shown in Figure 7(b), but the curves of the three warp-knitted spacers are basically the same. The Poisson's ratio e of the warp-knitted

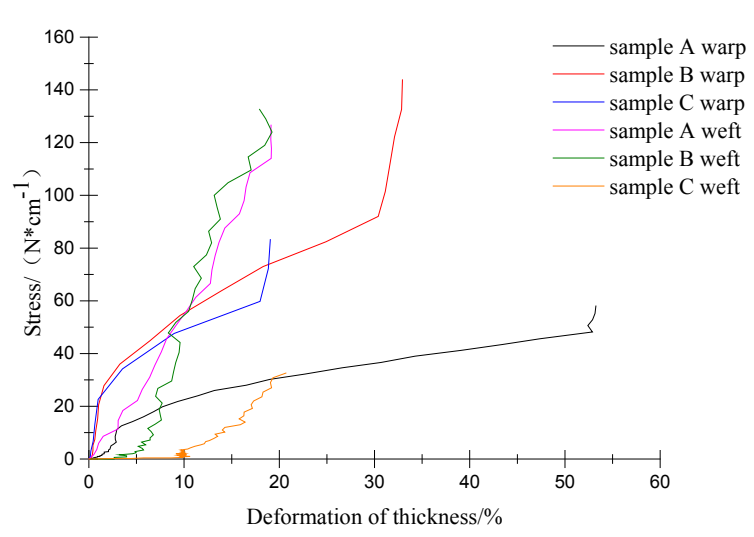

Figure 6. Stress-strain relations of deformation of samples in the thickness direction

(a)

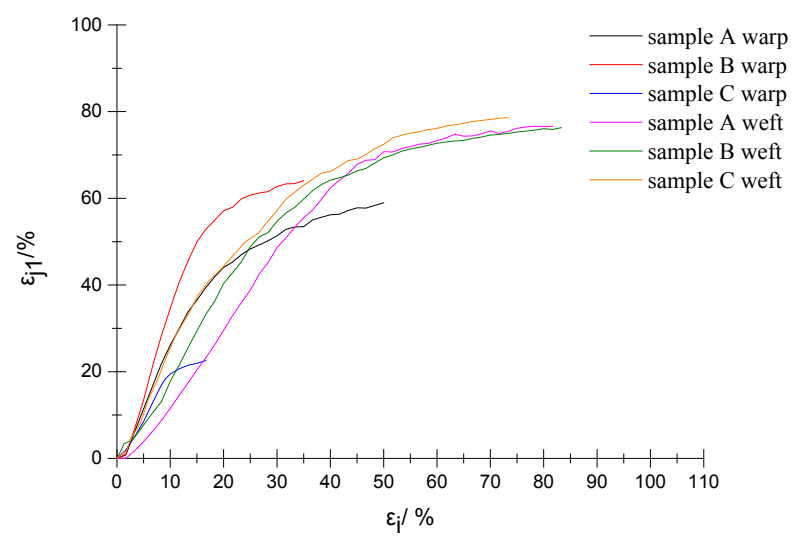

(b)

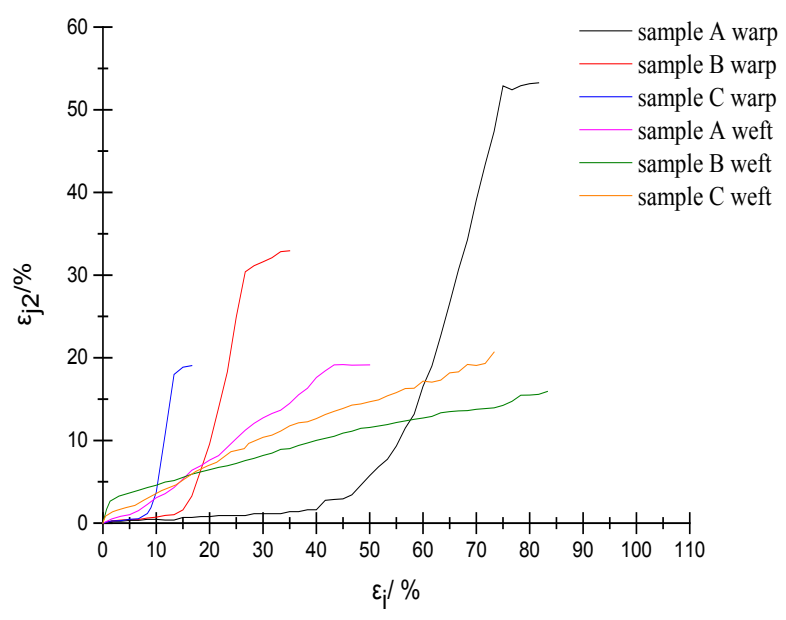

Figure 7. Poisson's ratio curve of samples: (a) Poisson's ratio curve of sample's surface layer; (b) Poisson's ratio curve of sample's interval layer

fabric surface layer is always greater than 1.0 in the initial stage. This is an indication that the shrinkage of the spacer fabric surface layer perpendicular to the direction of the force is greater than the amount of elongation in the direction of the force.

This is mainly related to the characteristics of the loop structure of the warp-knitted fabric. When the fabric is stretched in 
the warp direction or vice versa, the weft or warp yarns are extended, resulting in lateral deformation. At the end of the stretch, the fabric becomes tight, stifling further deformation. The size of the deformation does not change, so the surface layer's Poisson's ratio gradually becomes smaller.

The Poisson's ratio of the thickness section is $<1$ in the initial stage of stretching, indicating that the shrinkage in the thickness section of the spacer fabric is less than the amount of elongation in the direction of the force. At the later stages of stretching, the change of the Poisson's ratio is related to the stretching direction. The deformation in the thickness direction is always smaller than that in the stretching direction. During the warp-wise stretching process, it gets to a stage where deformation of the thickness section becomes greater than that in the warp-stretching direction. The curve exhibits a smaller slope segment.

\section{Conclusion}

This research report analyzed the 3D deformation orientation in the tensile state by proposing a new test method.

The deformation of the fabric in response to tensile stress in the vertical direction shows a characteristic initial large deformation, followed by minimal-to-no deformation. The fineness of the yarn is the main factor that affects the fabric breakage. Some fabrics are easily deformed in the warp-ward direction, and some are more easily weft-deformed.

The stress-strain curve in the thickness direction of warpknitted spacers is related to the stretching direction. In the latitudinal tension state, the spacer yarns in the thickness direction are not directly subjected to tension, and the curves show approximately linear changes coupled with a small strain. In the warp-wise-stretched state, the spacer yarns in the thickness section are affected by two surface forces and showed the "large stress with small deformation, then small stress with large deformation" feature.

The amount of shrinkage of the surface layer of the warpknitted fabric is larger than that in the direction of the force, and the amount of shrinkage in the thickness direction is smaller than the amount of elongation in the direction of tension. At the later stages of stretching, the change of Poisson's ratio is related to the direction of stretching.

\section{Acknowledgments}

The authors thank the Standards and Modes of Intelligent Manufacturing, Ministry of Industry and Information Technology, People's Republic of China (No. 1064130201160660); National Science Foundation of China (No. 61602212); Innovation fund project of Cooperation among Industries, Universities \& Research Institutes (CIUI), Jiangsu Province (No. BY201602234 ); and a project funded by the Priority Academic Program Development (PAPD) of Jiangsu Higher Education Institutions.

\section{References}

[1] Rajan, T. P., Souza, L. D., Ramakrishnan, G., Mohamed Zakriya, G. (2016). Comfort properties of functional warpknitted polyester spacer fabrics for shoe insole applications. Journal of Industrial Textiles, 45(6), 1239-1251.

[2] Xiaohua, Y., Hong, H., Xunwei, F. (2008). Development of the warp knitted spacer fabrics for cushion applications, Journal of Industrial Textiles, 37(3), 213-223.

[3] Yip, J., Ng, S. -P. (2008). Study of three-dimensional spacer fabrics. Journal of Materials Processing Technology, 206(1), 359-364.

[4] Xu-hong, M., Ming-Qiao, G. (2008). The compression behaviour of warp knitted spacer fabric. Fibres Text East Europe, 16(1), 90-92.

[5] Du, Z., Wu, Y., Li M., He, L. (2015). Analysis of structure of warp-knitted spacer fabric on pressure indices. Fibers and Polymers, 16(11), 2491.

[6] Liu, Y., Hu, H. (2015). Compressive mechanics of warpknitted spacer fabrics. Part II: a dynamic model. Textile Research Journal, 85(19), 2020-2029.

[7] Xuhong, M., Xiangyong, K., Gaoming, J. (2013). The experimental research on the stab resistance of warpknitted spacer fabric. Journal of Industrial Textiles, 43(2), 281-301.

[8] Du, Z., Yu, W., Hamada, H., Yang, Y. (2011). Analysis of structure and bending property of spacer fabric composites. Industria Textila, 62(2), 64-71.

[9] Dolatabadi, M. K., Kovar, R. (2012). Anisotropy in tensile properties of plain weave fabric-Part I: the meso-scale model. Textile Research Journal, 82(16), 1666-1676.

[10] Klevaitytè, R., Masteikaité, V. (2008). Anisotropy of woven fabric deformation after stretching. Fibres \& Textiles in Eastern Europe, 16(4), 69.

[11] King, M., Jearanaisilawong, P., Socrate, S. (2005). A continuum constitutive model for the mechanical behavior of woven fabrics. International Journal of Solids and Structures, 42(13), 3867-3896.

[12] Kim, H. -s., Park, C. H., (2016). Effect of biaxial tensile extension on superhydrophobicity of rayon knitted fabrics. RSC Advances, 6(53), 48155-48164.

[13] Yamada, T., Ito, N., Matsuo, M. (2003). Mechanical properties of knitted fabrics under uniaxial and strip biaxial extension as estimated by a linearizing method. Textile Research Journal, 73(11), 985-997.

[14] Dimitrovski, K. (2008). Influence of weave and weft characteristics on tensile properties of fabrics. Fibres \& Textiles in Eastern Europe, 16(2), 67.

[15] Sun, H., Pan, N., Postle, R. (2005). On the Poisson's ratios of a woven fabric. Composite Structures, 68(4), 505-510.

[16] Ma, P., Chang, Y., Boakye, A., Jiang, G. (2017). Review on the knitted structures with auxetic effect. The Journal of The Textile Institute, 108(6), 947-961.

[17] Hursa, A., Rolich, T., Ražić, S. E. (2009). Determining pseudo Poisson's ratio of woven fabric with a digital image correlation method. Textile Research Journal, 79(17), 1588-1598. 